\title{
Dynamic Texture Extraction And Video Denoising
}

\author{
Mathieu Lugiez $^{12}$, Michel Ménard ${ }^{1}$, and Abdallah El-Hamidi ${ }^{2}$ \\ 1 L3i - Université de La Rochelle \\ 2 MIA - Université de La Rochelle
}

\begin{abstract}
According to recent works, introduced by Y.Meyer [1] the decomposition models based on Total Variation (TV) appear as a very good way to extract texture from image sequences. Indeed, videos show up characteristic variations along the temporal dimension which can be catched in the decomposition framework. However, there are very few works in literature which deal with spatio-temporal decompositions. Thus, we devote this paper to spatio-temporal extension of the spatial color decomposition model. We provide a relevant method to accurately catch Dynamic Textures (DT) present in videos. Moreover, we obtain the spatio-temporal regularized part (the geometrical component), and we distinctly separate the highly oscillatory variations, (the noise). Furthermore, we present some elements of comparison between several models in denoising purpose.
\end{abstract}

\section{Introduction}

Decomposing an image into meaningful components appears as one of major aims in recent development in image processing. The first goal was image restoration and denoising; but following the ideas of Yes Meyer [1], in the Total Variation minimization framework of L. Rudin, S. Osher and E. Fatemi [2], image decomposition into geometrical and oscillatory components grants access to the textured part of an image, rejected by the previous model. We only cite, among many others, most recent works which we appear like most relevant and useful papers. In this way, reader can refer to the work of Aujol et al. [3] [4], Aujol and Chambolle [5], Aujol and Kang [6], Vese and Osher [7], Gilles [8] and more recently Bresson and Chan [9], Duval et al. [10] and Aubert et al. [11].

We are aiming to decompose videos into three components, regular one, the geometry $\mathbf{u}$, oscillating one $\mathbf{v}$, representative of texture and highly oscillating one, the noise $\mathbf{w}$ (see Fig.1 for a grayscale image decomposition example). So, we deal with color image sequences in extending to time existing reliable models. Moreover, through the texture component, we attempt to determinate the dynamics present in sequences, which will be suited for future work on Dynamic Texture (DT).

After have introduced different minimization functionals and a DT definition, we present the color spatio-temporal decomposition framework and subsequently their implementation. Then, we show some significant results, in particular the dynamicity of DT and comparing the denoising ability of the different models. 


\subsection{Decomposition models}

In 2001, Y. Meyer in [1] has proposed an extension of the famous image restoration model proposed by Rudin, Osher and Fatemi [2], based on the $B V^{3}$ space:

$$
\inf _{u \in B V} F^{R O F}(u)\left(J(u)+\frac{1}{2 \lambda}\|f-u\|_{L^{2}}^{2}\right)
$$

where $f$ is the original image and the parameter $\lambda$ controls the $L_{2}$-norm of the residual part $f-(u+v+w)$.

He has suggested an appropriate space $\left(G^{4}\right)$ to model oscillating signals (i.e texture and noise), close to the dual space of $B V$. Aujol et al. in [3] solve the Meyer algorithm, and propose the following minimization functional:

$$
\inf _{(u, v) \in B V \times G / f=u+v} F^{A 2 B C}(u, v)\left(J(u)+J^{*}(u)+\frac{1}{2 \lambda}\|f-u-v\|_{L^{2}}^{2}\right)
$$

where $J^{*}$ is the Legendre-Fenchel transform ${ }^{5}$ of $J$, so $J$ is the indicator function on $G_{\eta}=v \in G /\|v\|_{G} \leq \eta^{6}$.

To decompose image sequences in suitable components we propose to extend the Aujol-Chambolle [5] decomposition model. It rely on dual norms derived from $B V, G$ and $E^{7}$ spaces. Authors propose to minimize the following discretized functional:

$$
\begin{aligned}
\inf _{(u, v, w) \in X^{3} / f=u+v+w} F^{A C}(u, v, w)= & \underbrace{J(u)}_{\begin{array}{c}
\text { Regularization: } \\
\text { TV }
\end{array}}+\underbrace{J^{*}\left(\frac{v}{\eta}\right)}_{\begin{array}{c}
\text { Texture } \\
\text { extraction }
\end{array}}+\underbrace{B^{*}\left(\frac{w}{\delta}\right)}_{\begin{array}{c}
\text { Noise extraction by } \\
\text { wavelet shrinkage }
\end{array}} \\
& +\underbrace{\frac{1}{2 \lambda}\|f-u-v-w\|_{X}^{2}}_{\text {Residual part }}
\end{aligned}
$$

where $X$ is the Euclidian space $\mathbb{R}^{N \times N}$. $J^{*}$ and $B^{*}$, are the Legendre-Fenchel transform of respectively $J$ and $B$. See Fig.1 for an image decomposition example through $F^{A C}$.

\footnotetext{
${ }^{3} B V(\Omega)$ is the subspace function $u \in L^{1}(\Omega)$ such that the following quantity, the total variation of $u$, is finite: $J(u)=\sup \left\{\int_{\Omega} u(x) \operatorname{div}(\xi(x)) d x\right\}$ such that $\xi \in C_{c}^{1}\left(\Omega, \mathbb{R}^{2}\right),\|\xi\|_{L^{\infty}(\Omega)} \leq 1$

$4 G$, is a Banach space, composed of all distributions $v$ : $v=\partial_{1} g_{1}+\partial_{2} g_{2}=\operatorname{div}(g)$ with $g_{1}$ and $g_{2} \in L^{\infty}\left(\mathbb{R}^{2}\right)$ endowed with the following norm $\|v\|_{G}=\inf \left\{\|g\|_{L^{\infty}\left(\Omega, \mathbb{R}^{2}\right)} / v=\operatorname{div}(g), g=\right.$ $\left.\left(g_{1}, g_{2}\right),|g(x)|=\sqrt{\left(\left|g_{1}\right|^{2}+\left|g_{2}\right|^{2}\right)(x)}\right\}$

$5 F^{*}(v)=\sup _{u}\left(\langle u, v\rangle_{L^{2}}-F(u)\right)$, where $\langle.,\rangle_{L^{2}}$ stands for the $L^{2}$ inner product.

6 i.e: $J^{*}\left(\frac{v}{\eta}\right)=0$ if $v \in G_{\eta},+\infty$ elsewhere

${ }^{7} E$ is a Banach space to model very oscillating patterns: $E=\dot{B}_{-1, \infty}^{\infty}$ dual space of $\dot{B}_{1,1}^{1}$.
} 


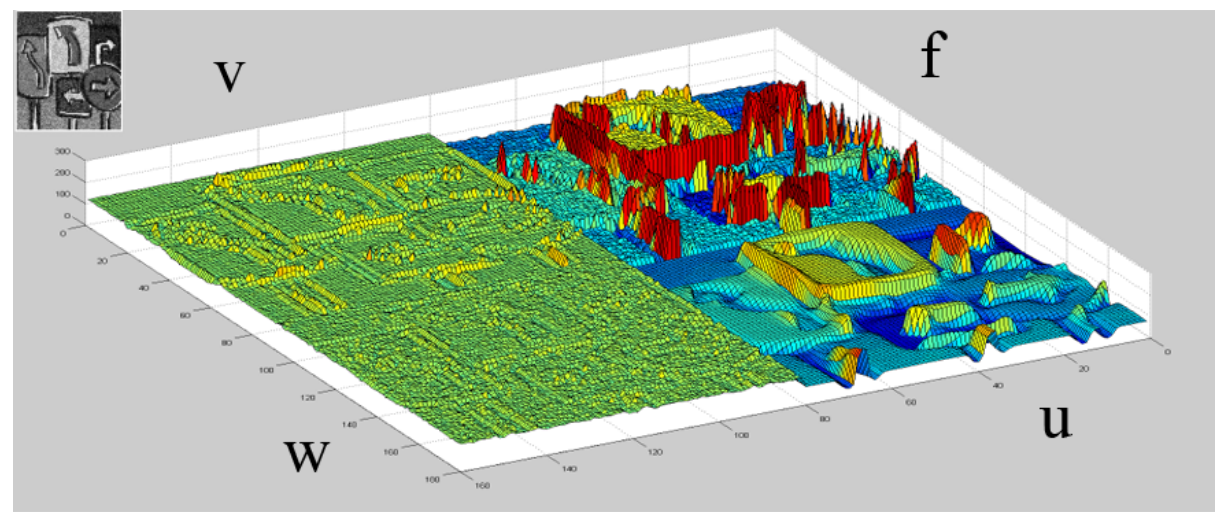

Fig. 1. Grayscale image decomposition with AC functional. From top to bottom and left to right: Texture component $\left(\mathbf{v}_{\mathrm{AC}}\right)$, original noisy image, noise component $\left(\mathbf{w}_{\mathrm{AC}}\right)$ and regular or geometrical part $\left(\mathbf{u}_{\mathrm{AC}}\right)$.

\subsection{Repetitivity in video and Dynamic Texture (DT)}

A new issue in texture analysis is its extension to the temporal dimension, a field known as Dynamic Texture Analysis. In DTA, the notion of self-similarity central to conventional image textures is extended to the spatio-temporal domain. DT typically results from processes such as water flows, smoke, fire, flag blowing in the wind or moving escalator. Important tasks are thus detection [12], segmentation [13], synthesization and perceptual characterization [14] of DT.

Image sequences present variations along time dimension. These variations could be, purely temporal (led blinking), spatial in movement (a leaf waving into wind like as shown in Fig.2:(1)), periodic or pseudo-periodic (like an escalator or the low frequencies dues to quasi periodic anemone contractions as shown in Fig.2:(3)) or spatio-temporal (flowing water and wavelets as shown in Fig.2:(2)). To properly catch all this spatio-temporal variation (i.e DT) and effectively separate them from spatio-temporal structures we consider a video as an pseudo 3 -D image, i.e a volume, so that we can apply 2-D image algorithms correctly extended to the $2-\mathrm{D}+\mathrm{t}$ case. Indeed, straight spatial (i.e frame by frame) point of view is not sufficient to rightly discern patterns presenting dynamic process or movement. Moreover since static informations are redundant in a sequence, and since we take into account temporal variations, we grant to decomposition process to be more accurate and efficient for discerning the geometry and texture.

\section{Spatio-temporal extension}

\subsection{Spatio-temporal structure and discretization}

We assume that we have a given image sequence $f \in L^{2}(\Omega)$, where $\Omega$ is an open and bounded domain on $\mathbb{R}^{3}$, with Lipschitz boundary. In order to recover $\mathbf{u}, \mathbf{v}$ and $\mathbf{w}$ from $\mathbf{f}$, we propose: 

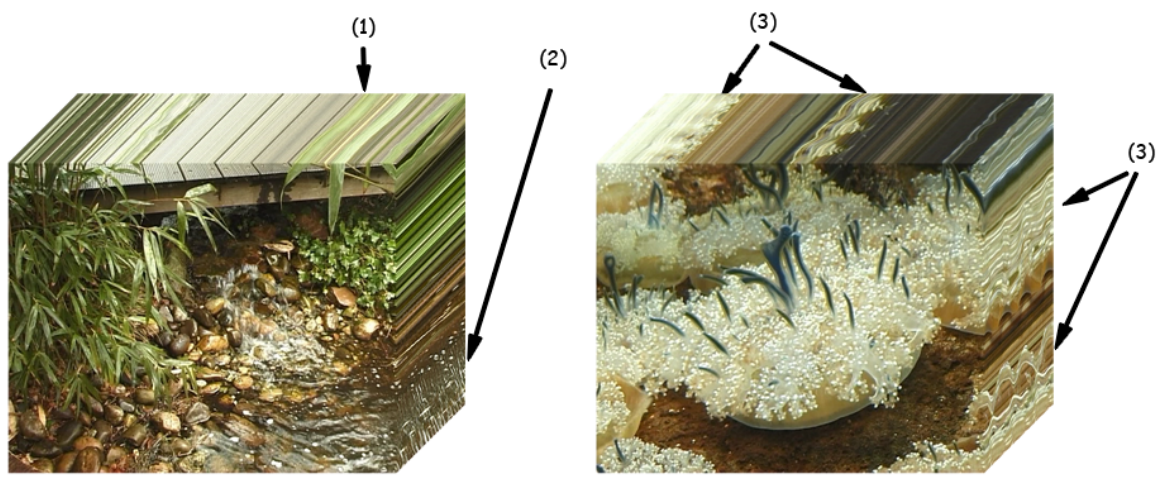

Fig. 2. 2D+t block of two dynamic textures. Here, a dynamic texture is seen as a data cube. One can clearly see at (1) leaves blowing in wind: a spatial texture in movement, at (2) flowing water and wavelets: spatio-temporal texture and at (3) spatio-temporal low frequencies dues to periodic anemone contractions.

- an extended discrete version of gradient vector: $|\nabla \mathbf{u}|_{x y t}$ given by:

$$
(\nabla \mathbf{u})_{i, j, k}=\left((\nabla \mathbf{u})_{i, j, k}^{x},(\nabla \mathbf{u})_{i, j, k}^{y},(\nabla \mathbf{u})_{i, j, k}^{t}\right)
$$

where,

$$
(\nabla \mathbf{u})_{i, j, k}^{t}= \begin{cases}\mathbf{u}_{i, j, k+1}-\mathbf{u}_{i, j, k-1} & \text { if } k<N \\ 0 & \text { if } k=1 \text { or } N\end{cases}
$$

\section{- an extended discrete total variation definition:}

$$
J(\mathbf{u})=\int_{\Omega} \sqrt{\sum_{c=R, G, B}\left|\left((\nabla \mathbf{u})_{i, j, k}^{x}\right)_{c}\right|^{2}+\left|\left((\nabla \mathbf{u})_{i, j, k}^{y}\right)_{c}\right|^{2}+\alpha\left|\left((\nabla \mathbf{u})_{i, j, k}^{t}\right)_{c}\right|^{2} d x d y d t}
$$

We introduce the constant $\alpha$ to maintain homogeneity between space and time components. The quantization steps can be different along space and time dimensions. In practice, we often set it to one, but user can tune it.

- an adapted definition of $G$ : inspired by [7] for the vectorial $R G B$ case, extended to the third dimension:

Definition 1. Let $G$ denote the Banach space consisting of all generalized vectorvalued functions $\overrightarrow{\boldsymbol{v}}(x, y, t)=\left(\boldsymbol{v}_{R}(x, y, t), \boldsymbol{v}_{G}(x, y, t), \boldsymbol{v}_{B}(x, y, t)\right)$ which can be written as:

$$
\begin{aligned}
\overrightarrow{\boldsymbol{v}}(x, y, t) & =\left(\operatorname{div} \overrightarrow{\boldsymbol{g}_{R}}, \operatorname{div} \overrightarrow{\boldsymbol{g}_{G}}, \operatorname{div} \overrightarrow{\boldsymbol{g}_{B}}\right) \\
\boldsymbol{g}_{x, c}, \boldsymbol{g}_{y, c}, \boldsymbol{g}_{t, c} & \in L^{\infty}\left(\mathbb{R}^{3}\right), c=R, G, B
\end{aligned}
$$


induced by the norm $\|\boldsymbol{v}\|_{*}$ defined as the lower bound of all $L^{\infty}$ norms of functions $|\overrightarrow{\boldsymbol{g}}|$, where $|\overrightarrow{\boldsymbol{g}}|=\sqrt{\left|\overrightarrow{\boldsymbol{g}_{R}}\right|^{2}+\left|\overrightarrow{\boldsymbol{g}_{G}}\right|^{2}+\left|\overrightarrow{\boldsymbol{g}_{B}}\right|^{2}}=\sqrt{\sum_{c=R, G, B}\left(\left(\boldsymbol{g}_{x, c}\right)^{2}+\left(\boldsymbol{g}_{y, c}\right)^{2}+\left(\boldsymbol{g}_{t, c}\right)^{2}\right)}$, and where the infinitum is computed over all decompositions (6) of $\overrightarrow{\boldsymbol{v}}$.

- an adapted shrinkage scheme: In [15], authors use a connection given by Weickert in [16] between wavelet shrinkage and non linear diffusion filtering to introduce the noise component in color image decomposition model. Indeed, by considering an explicit discretization and relating it to wavelet shrinkage, Weickert gives shrinkage rules where all channels are coupled. The formula states a general correspondence between a shrinkage function $W_{S T}\left(S_{\theta}\left(w_{j}^{i}, \delta\right)\right)$ and the total variation diffusivity of an explicit nonlinear diffusion scheme. So, in order to steer the evolution of all three channels in spatio-temporal domain, the following shrinkage function $W_{S T}$, relying on weight of $S_{\theta}$ for the wavelet coefficient $w_{i}^{j}$, is proposed:

$$
S_{\theta}\left(w_{i}^{j}\right)=w_{i}^{j}\left(1-39 \theta\left(\sqrt{\sum_{i} c *\left(w_{R, i}^{j}\right)^{2}+c *\left(w_{G i}^{j}\right)^{2}+c *\left(w_{B i}^{j}\right)^{2}}\right)^{-1}\right)
$$

where $w_{j}^{i}$ is the wavelet coefficient, $j$ the resolution and $c=1$ if $i \in\{x, y, t\}$, $c=2$ if $i \in\{x y, x t, y t\}$ and $c=4$ if $i=x y t$. The soft wavelet thresholding functional $W_{S T}$ for a given wavelet coefficient, with threshold $\delta$ is given by:

$$
W_{S T}\left(S_{\theta}\left(w_{i}^{j}\right), \delta\right)= \begin{cases}S_{\theta}\left(w_{i}^{j}\right)-\delta & \text { if } S_{\theta}\left(w_{i}^{j}\right) \geq \delta \\ 0 & \text { if }\left|S_{\theta}\left(w_{i}^{j}\right)\right| \leq \delta \\ S_{\theta}\left(w_{i}^{j}\right)+\delta & \text { if } S_{\theta}\left(w_{i}^{j}\right) \leq-\delta\end{cases}
$$

\subsection{Numerical implementation}

Thanks to recent advances in color image processing ([10] and [9]), motivating by the well known Chambolle's projection [17], we are able now to use a projected gradient algorithm computing the solution of this dual formulation problem extended to time. So we dispose of an efficient way to numerically solve the different minimization problem induced by the extended functional (3), using fixed point method: $\mathbf{P}^{0}=0$, and

$$
\mathbf{P}_{i, j, k}^{n+1}=\frac{\mathbf{P}_{i, j, k}^{n}+\tau\left(\nabla\left(\operatorname{div}\left(\mathbf{P}^{n}\right)-\frac{\mathbf{f}}{\lambda}\right)\right)_{i, j, k}}{\max \left(1,\left|\mathbf{P}^{n}+\tau\left(\nabla\left(\operatorname{div}\left(\mathbf{P}^{n}\right)-\frac{\mathbf{f}}{\lambda}\right)\right)_{i, j, k}\right|_{2}\right)}
$$

with $\tau<\frac{\lambda}{\eta}$ to ensure the convergence of the algorithm [9].

In order to compute the solution of problem (1) we propose the following numerical scheme adapted to the vectorial formulation (Eq.(4) - (9)) according to spatio-temporal point of view:

$$
\begin{aligned}
& \mathbf{u}_{\mathrm{ROF}}=\mathbf{f}-P_{G_{\lambda}}(\mathbf{f}) \\
& \mathbf{v}_{\mathrm{ROF}}=P_{G_{\lambda}}(\mathbf{f})
\end{aligned}
$$


To solve the problem (2), following Aujol et al. [3], we adapted the solution to the vectorial and spatio-temporal (Eq.(4) - (9)) case and propose the following algorithm:

1. Initialization: $\mathbf{u}_{0}=0, \mathbf{v}_{0}=0$

2. Iterations:

$$
\begin{aligned}
\tilde{\mathbf{v}}_{\mathrm{A} 2 \mathrm{BC}} & =P_{G_{\eta}}\left(\mathbf{f}-\mathbf{u}_{\mathrm{A} 2 \mathrm{BC}}\right) \\
\tilde{\mathbf{u}}_{\mathrm{A} 2 \mathrm{BC}} & =\mathbf{f}-\mathbf{u}_{\mathrm{A} 2 \mathrm{BC}}-\tilde{\mathbf{v}}_{\mathrm{A} 2 \mathrm{BC}}-P_{G_{\lambda}}\left(\mathbf{f}-\tilde{\mathbf{v}}_{\mathrm{A} 2 \mathrm{BC}}\right)
\end{aligned}
$$

3. Repeated until:

$$
\max \left(\left|\tilde{\mathbf{u}}_{\mathrm{A} 2 \mathrm{BC}}-\mathbf{u}_{\mathrm{A} 2 \mathrm{BC}}\right|,\left|\tilde{\mathbf{v}}_{\mathrm{A} 2 \mathrm{BC}}-\mathbf{v}_{\mathrm{A} 2 \mathrm{BC}}\right|\right) \leq \epsilon
$$

So, to resolve the minimization problem (3) we propose to follow the numerical scheme of Aujol and Chambolle [5] adapted to vectorial formulation (Eq.(4) - (9)):

1. Initialization: $\mathbf{u}_{0}=0, \mathbf{v}_{0}=0, \mathbf{w}_{0}=0$

2. Iterations:

$$
\begin{aligned}
\tilde{\mathbf{w}}_{\mathrm{AC}} & =P_{\delta B_{E}}\left(\mathbf{f}-\mathbf{u}_{\mathrm{AC}}-\mathbf{v}_{\mathrm{AC}}\right) \\
& =\mathbf{f}-\mathbf{u}_{\mathrm{AC}}-\mathbf{v}_{\mathrm{AC}}-W_{S T}\left(\mathbf{f}-\mathbf{u}_{\mathrm{AC}}-\mathbf{v}_{\mathrm{AC}}, \delta\right) \\
\tilde{\mathbf{v}}_{\mathrm{AC}} & =P_{G_{\eta}}\left(\mathbf{f}-\mathbf{u}_{\mathrm{AC}}-\tilde{\mathbf{w}}_{\mathrm{AC}}\right) \\
\tilde{\mathbf{u}}_{\mathrm{AC}} & =\mathbf{f}-\mathbf{u}_{\mathrm{AC}}-\tilde{\mathbf{v}}_{\mathrm{AC}}-\tilde{\mathbf{w}}_{\mathrm{AC}}-P_{G_{\lambda}}\left(\mathbf{f}-\tilde{\mathbf{v}}_{\mathrm{AC}}-\tilde{\mathbf{w}}_{\mathrm{AC}}\right)
\end{aligned}
$$

3. Repeated until:

$$
\max \left(\left|\tilde{\mathbf{u}}_{\mathrm{AC}}-\mathbf{u}_{\mathrm{AC}}\right|,\left|\tilde{\mathbf{v}}_{\mathrm{AC}}-\mathbf{v}_{\mathrm{AC}}\right|,\left|\tilde{\mathbf{w}}_{\mathrm{AC}}-\mathbf{w}_{\mathrm{AC}}\right|\right) \leq \epsilon
$$

\section{Numerical Results: Static versus Dynamic Decomposition (SD vs DD)}

All images and results are computed from DynTex [18], a dynamic texture database which provides a diverse high-quality DT. DynTex sequences come from natural scenes presenting a wide variety of moving process. Such diversity grants user to identify and emphasize a lot of aspects in testing purpose.

\subsection{Dynamic Texture Extraction}

To analyze the influence of the dynamic property of the texture we present a comparison between the two methods of decomposition, SD versus DD, (both computed with same parameters). We can easily see that time impact in result: water in Fig.3 is well regularized and fluid aspect is well represent into the $\mathbf{v}_{\mathrm{AC}}$ component. The regularization is more robust to illumination and movement constraints. 


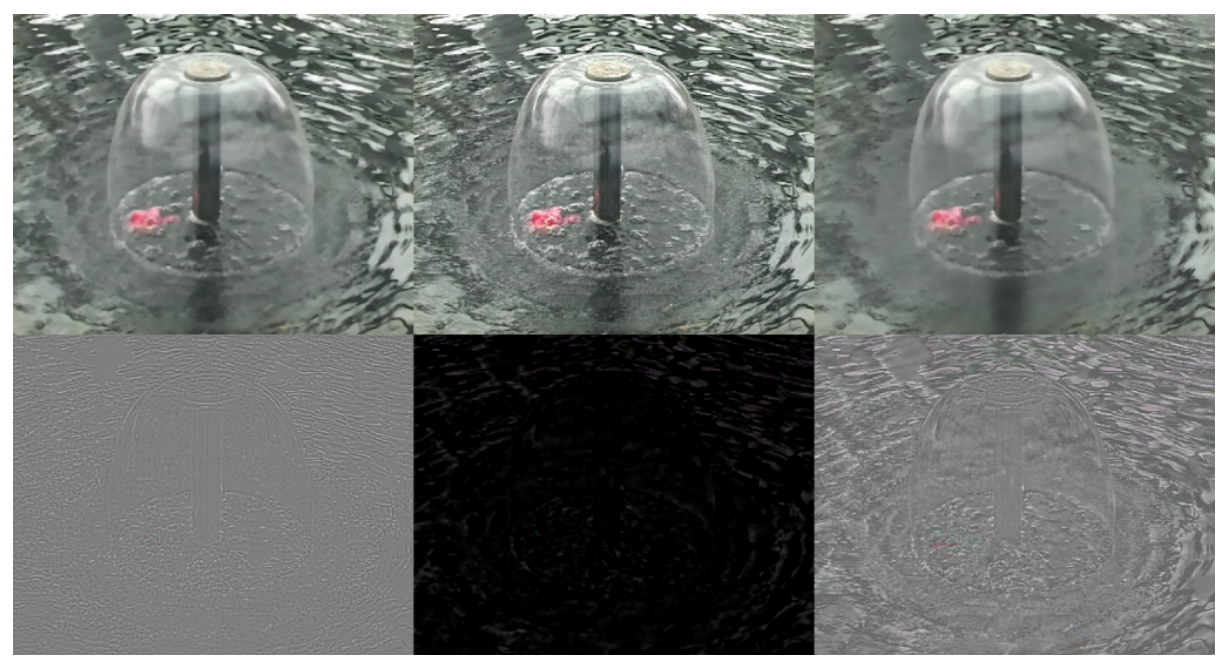

Fig. 3. Left: the geometrical component, $\mathbf{u}_{\mathrm{AC}}$, in SD (top) and its texture component $\mathbf{v}_{\mathrm{AC}}$ (bottom). Center and top: the original image, bottom: Difference between the static and dynamic $\mathbf{v}_{\mathrm{AC}}$ components. Right: the geometrical component in DD (top) and its dynamic texture $\mathbf{v}_{\mathrm{AC}}$ (bottom).

Moreover, if user tunes parameters to obtain a stronger regularization, our algorithm is able to catch wider waves into spatio-temporal texture component: see around the circumference of fountain in Fig.3, small wavelets are well catched in $\mathbf{v}_{\mathrm{AC}}$ than wider waves. It's a matter of deep in spatio-temporal texture extraction, which our algorithm is able to deal with, like shown in Fig.4.

We present, in Fig.5, a part of a decomposed sequence of flowing water under wood bridge. We can see the static aspect of $\mathbf{u}_{\mathrm{AC}}$ component, regularized in space and in time. It seems to be freezed, although texture component, $\mathbf{v}_{\mathrm{AC}}$, present a real dynamic. Only moving things or objects presenting dynamicity are taken in account in the temporal divergence part of $\mathbf{v}_{\mathrm{AC}}$ component.

The third part of texture component play a key role in our process, movement information is well captured. In this way we obtain the dynamicity present in video through oscillations along time dimension. These results will be useful for future work on dynamic texture characterization. 


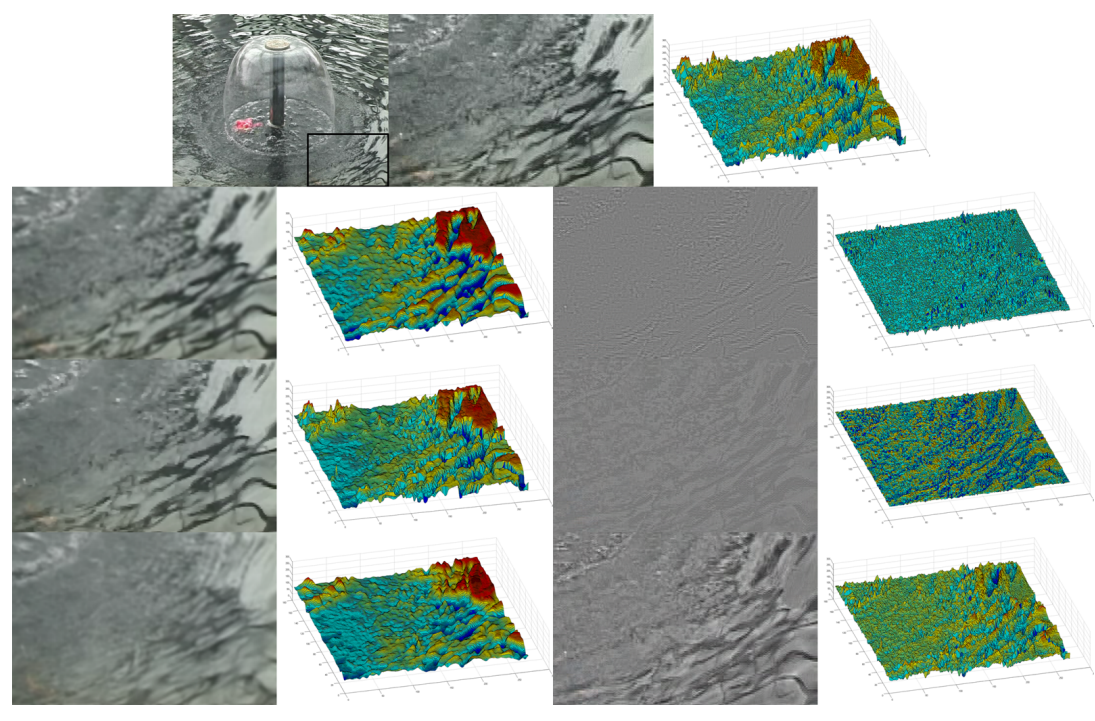

Fig. 4. First row: original image from sequence 6487310 of DynTex, the ROI and a visualization of ROI. Second row: SD of ROI with $\lambda=140, \eta=140$ from left to right $\mathbf{u}_{\mathrm{AC}}$ component and its visualization, since $\mathbf{v}_{\mathrm{AC}}$ component and its visualization. Third row, DD of the ROI with $\lambda=10$ and $\eta=10$ (weak regularization and texture extraction). Fourth row, DD of the ROI with $\lambda=140$ and $\eta=140$ (strong regularization and texture extraction). We can see than SD is not able to deal with dynamicity, moreover, in DD user can catch different scales of waves in the decomposition process.

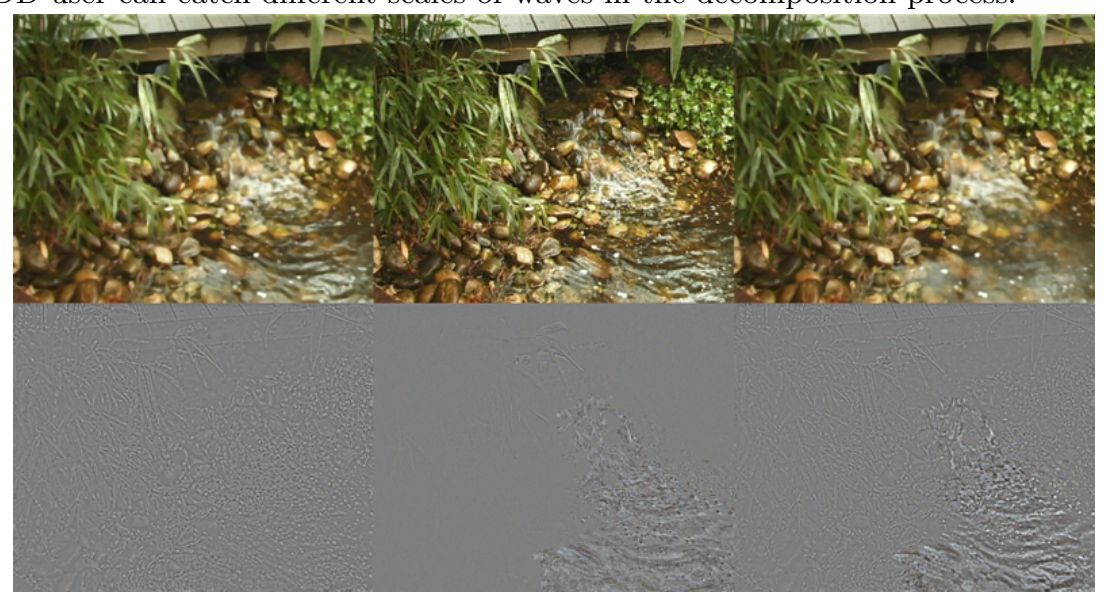

Fig. 5. From left to right, the geometrical component, $\mathbf{u}_{\mathrm{AC}}$, in classic color decomposition (top) and its texture and noise component $\mathbf{v}_{\mathrm{AC}}$ (bottom). The original image (center and top), the time influence in calculus of divergence of $\mathbf{v}_{\mathrm{AC}}$ component (center and bottom). Then our new dynamic decomposition components (right). 


\section{$3.2 \quad$ Video denoising}

We present in this section some elements of comparison between the different models in denoising purpose. Fig. 6 to 9 present the decomposition of one image, pick up from a sequence of eight images, noised separately (and decomposed conjointly in the DD process). We present the result of denoising process of the different models. One can easily see that DD clearly outperform SD whatever the model. Moreover the AC functional give the better results even if the texture component catch a part of noise in the decomposition process.
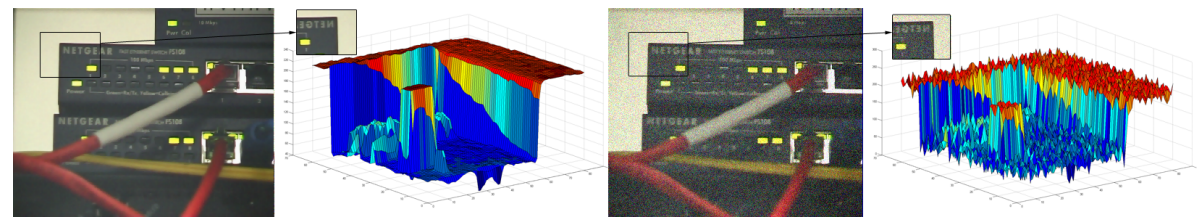

Fig. 6. From left to right, an original image from the sequence 64 cc610 of DynTex, the noised version (gaussian noise with void mean and $\sigma=20, \mathrm{SNR}=14.53, \mathrm{PSNR}=21.18$ ), and a visualization of ROI.
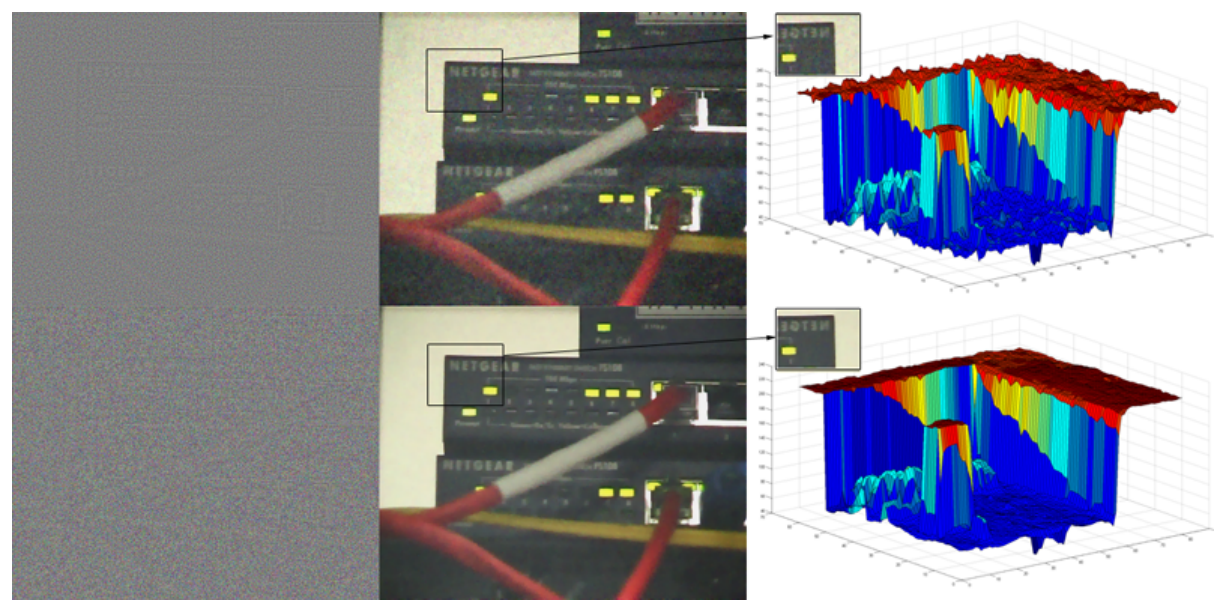

Fig. 7. ROF decomposition with $\lambda=25$. Top: SD, bottom: DD. Left the vrof component, center uROF and right a visualization of ROI. We have a SNR equal to 23.03 and a PSNR equal to 29.54 with SD and respectively 29.09 and 35.59 with DD.

In the case of very noisy sequence, $(\sigma=50, \mathrm{SNR}=10.85$ and PSNR=17.49), computed with exactly the same parameters and the same sequence, we have: 

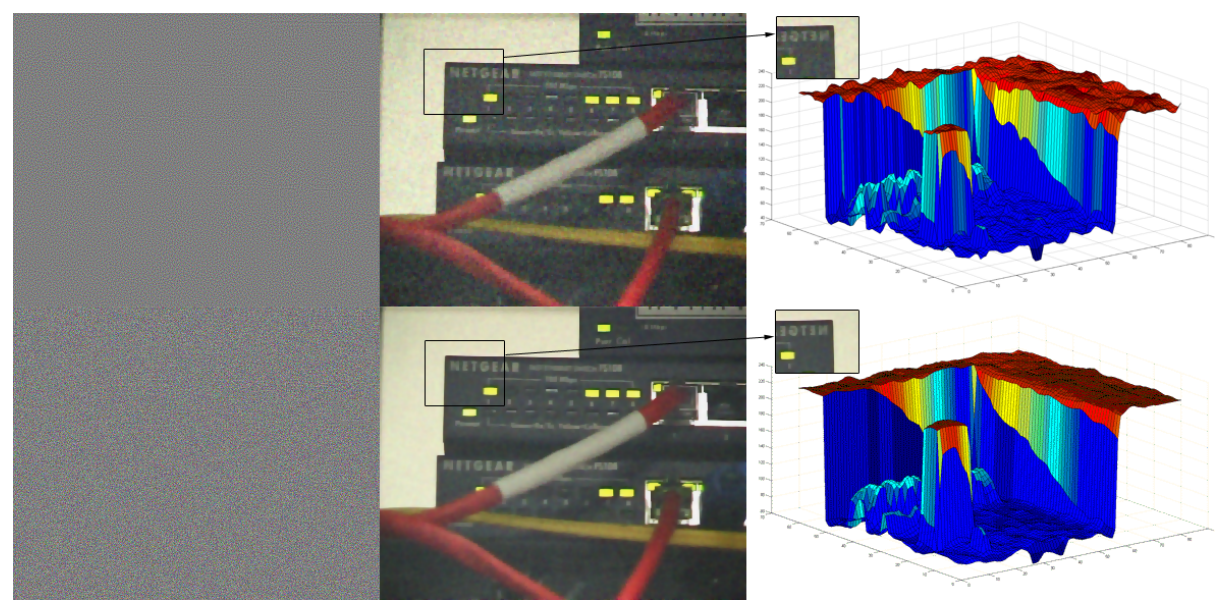

Fig. 8. A2BC decomposition with $\lambda=10$ and $\eta=25$. Top: SD, bottom: DD. Left the $\mathbf{v}_{\mathrm{A} 2 \mathrm{BC}}$ component, center $\mathbf{u}_{\mathrm{A} 2 \mathrm{BC}}$ and right a visualization of ROI. We have a SNR equal to 24.56 and a PSNR equal to 31.12 with SD and respectively 29.57 and 36.08 with DD.
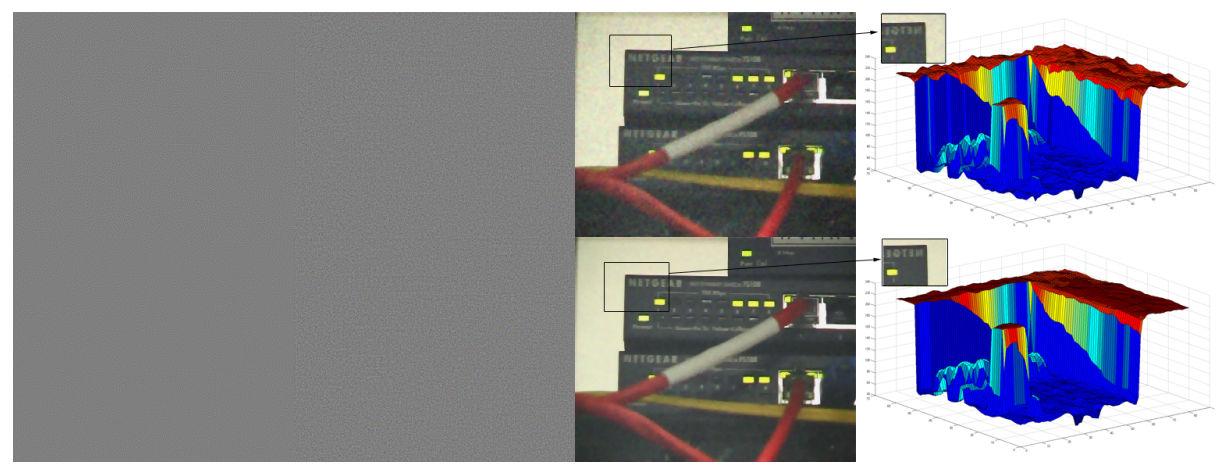

Fig. 9. AC decomposition with $\lambda=10, \eta=15$ and $\delta=45$. Top: SD, bottom: DD. Left the $\mathbf{v}_{\mathrm{AC}}$ component, center $\mathbf{u}_{\mathrm{AC}}$ and right a visualization of ROI. We have a SNR equal to 25.10 and a PSNR equal to 31.60 with SD and respectively 30.24 and 36.74 with DD.

- ROF: for SD we obtain a $\mathrm{SNR}=16.40$ a PSNR=22.90 and for DD we have respectively 21.04 and 27.55 .

- A2BC: for SD we obtain a $\mathrm{SNR}=18.32$ a $\mathrm{PSNR}=24.83$ and for DD we have respectively 25.23 and 31.73 .

- AC: for SD we obtain a $\mathrm{SNR}=20.58$ a $\mathrm{PSNR}=27.12$ and for DD we have respectively 26.91 and 33.42 . 


\section{Discussion}

We have investigate in the present paper the spatio-temporal extension of color decomposition models through projection approach. One can see that taking into account variations along the temporal axe appears as a useful and innovative way to catch the dynamic texture present in videos. Moreover this extension provide a useful tool for video denoising, indeed dynamic model clearly outperform classical decomposition, reinforcing the denoising ability of $T V$ based models. So, we provide an efficient tool to separate dynamic texture, structure and noise in image sequence.

\section{References}

1. Meyer, Y.: Oscillating patterns in image processing and nonlinear evolution equations. The fifteenth dean jacqueline B. Lewis Memorial Lectures (2001)

2. L.Rudin, S.Osher, E.Fatemi: Nonlinear total variation based noise removal. Physica D 60 (1992) 259-269

3. Aujol, J.F., Aubert, G., Blanc-Féraud, L., Chambolle, A.: Image decomposition into a bounded variation component and an oscillating component. Journal of Mathematical Imaging and Vision 22(1) (2005) 71-88

4. Aujol, J.F., Gilboa, G., Chan, T., Osher, S.: Structure-texture image decomposition - modeling, algorithms, and parameter selection. International Journal of Computer Vision 67(1) (2006) 111-136

5. Aujol, J.F., Chambolle, A.: Dual norms and image decomposition models. International Journal of Computer Vision 63(1) (2005) 85-104

6. Aujol, J.F., Kang, S.H.: Color image decomposition and restoration. J. Visual Communication and Image Representation 17(4) (2006) 916-928

7. Vese, L.A., Osher, S.: Color texture modeling and color image decomposition in a variational-PDE approach. In: SYNASC, IEEE Computer Society (2006) 103-110

8. Gilles, J.: Noisy image decomposition: A new structure, texture and noise model based on local adaptivity. J. Math. Imaging Vis. 28(3) (2007) 285-295

9. Bresson, X., Chan., T.: Fast minimization of the vectorial total variation norm and applications to color image processing. In: SIAM Journal on Imaging Sciences (SIIMS). (submitted 2007)

10. Duval, V., Aujol, J.F., Vese, L.: A projected gradient algorithm for color image decomposition. Technical report, CMLA Preprint 2008-21 (2008)

11. Aubert, G., El-Hamidi, A., Ghannam, C., Ménard, M.: On a class of ill-posed minimization problems in image processing. Journal of Mathematical Analysis and Applications 352(1) (2009) 380 - 399 Degenerate and Singular PDEs and Phenomena in Analysis and Mathematical Physics.

12. Dedeoglu, Y., Toreyin, B.U., Gudukbay, U., Cetin, A.E.: Real-time fire and flame detection in video. In: IEEE International Conference on Acoustics, Speech, and Signal Processing (ICASSP'05). Volume Volume II. (2005) pages 669-673

13. Chetverikov, D., Péteri, R.: A brief survey of dynamic texture description and recognition. In Springer-Verlag., ed.: 4th International Conference on Computer Recognition Systems (CORES'05), "Advances in Soft Computing", Poland (2005) pages $17-26$ 
14. Péteri, R., Chetverikov., D.: Dynamic texture recognition using normal flow and texture regularity. In Springer, ed.: 2nd Iberian Conference on Pattern Recognition and Image Analysis (IbPRIA'05). Volume 3523 of Lecture Notes in Computer Science., Estoril, Portugal (2005) 223-230

15. Dubois, S., Lugiez, M., Péteri, R., Ménard, M.: Adding a noise component to a color decomposition model for improving color texture extraction. In: 4th European Conference on Colour in Graphics, Imaging, and Vision, Barcelona : Espagne (2008). (2008)

16. Weickert, J., Steidl, G., Mrazek, P., Welk, M., Brox, T.: Chapter 1. In: (Diffusion filters and wavelets: What can they learn from each other?)

17. Chambolle, A.: An algorithm for total variation minimization and its applications. JMIV 20 (2004) 8997

18. Péteri, R., Huiskes, M., Fazekas, S.: (Dyntex : A comprehensive database of dynamic textures) http://www.cwi.nl/projects/dyntex/. 